\title{
INTERACCIÓN ENTRE ABUSO DE ALCOHOL Y VIOLENCIA DOMÉSTICA. LAS ENFERMERAS PIEZA CLAVE 1
}

\author{
JOSÉ RAMÓN MARTÍNEZ RIERA \\ Licenciado en Enfermería por la Hogeschool Zeeland \\ Director de Enfermería A.P. Área 17. Elda (Alicante)
}

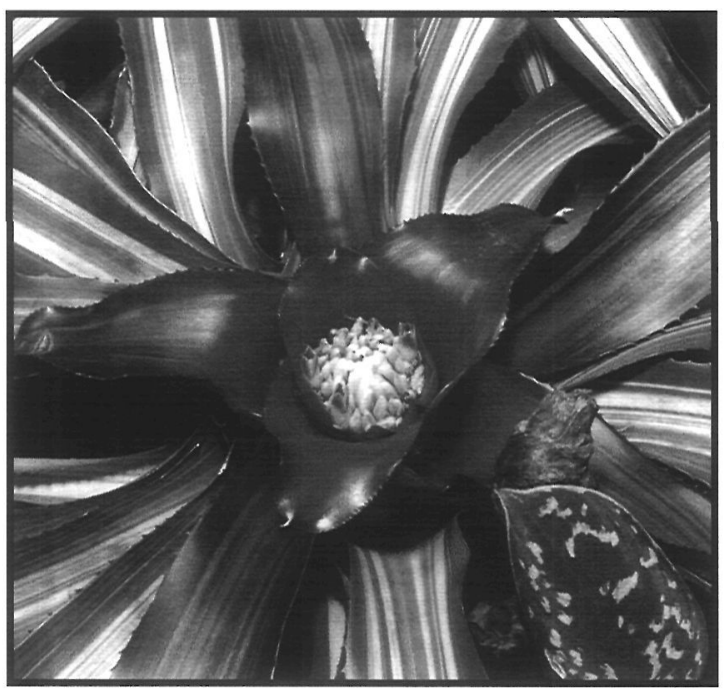

\section{RELATIONSHIIP BETWEEN ALCOHOL ABUSE AND DOMESTIC VIOLENCE 1}

Nurses, key role

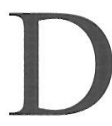
omestic violence has become one of the core problems in our society. Although various issues are involved, alcohol intake is one of the key triggering factors. The present article approaches this problem, focusing on the role that health professionals in general and nurses in particular play with regard to prevention and detection.
Interventions approached from a holistic perspective rather than a limited biologicist one, emphasise the need of getting involved in such a relevant subject.

The development of health programmes such as Menopause in our district, allows the detection of domestic violence warning signals through participation in activities that contribute to interaction between women and health professionals.

Key words: domestic violence, alcoholism, health professionals, nurses.

\section{RESUMEN}

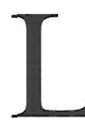

a violencia doméstica se ha convertido en uno de los principales problemas de salud de nuestra Sociedad. Son múltiples los factores que intervienen en la misma pero hay que destacar la interacción que tiene el alcoholismo como desencadenante de dicha violencia. En el presente artículo se hace una aproximación a este problema al tiempo que se destaca el papel de los servicios sanitarios en general y de las enfermeras, como profesionales de los mismos, en particular para su detección y prevención.

$\mathrm{El}$ abordaje desde una perspectiva integral alejada de la valoración biologicista y reduccionista de la intervención de los profesionales sanitarios

\footnotetext{
1 Adaptation of the paper presented during the course "Introduction to interaction between alcohol abuse and domestic violence", that took place in Alicante and Valencia (november 2001), organised by the Foundation Salud y Comunidad and the Dirección General de Drogodeeandencias, Consellería de Bienestar Social)

${ }_{1}^{1}$ Adaptación de la ponencia desarrollada en el Curso "Introducción a la Interacción entre abuso de Alcohol y Violencia doméstica" desarrollado en Valencia y Alicante en Noviembre y Diciembre de 2001, organizado por la Fundación "Salud y Comunidad y la Conselleria de Bienestar Social: Dirección General de Drogodependencias"
} 
pone de manifiesto la necesidad de implicarse en un tema de tanta trascendencia en el que el dilema cuidar - curar adquiere una especial importancia.

El desarrollo de determinados programas de Salud como el del climaterio desarrollado en el Área 17, que se expone, pueden servir para la detección de señales de alarma de violencia doméstica a través de actividades participativas que favorezcan la interacción entre los profesionales sanitarios y las mujeres que conduzcan a eliminar o al menos minimizar los efectos de este importante problema de salud comunitario.

Palabras clave: violencia doméstica, alcoholismo, profesionales sanitarios, enfermeras.

\section{IDENTIDAD DE GÉNERO}

Resulta, o puede resultar, paradójico que un hombre hable de violencia doméstica o de violencia de género cuando se trata de un problema sufrido fundamentalmente por las mujeres, generado y ejercido eminentemente por los hombres. En cualquier caso considero que la violencia, más allá del gramatical, no tiene género aunque los hombres nos hemos empeñado a lo largo de la historia en hacerla compañera habitual de nuestras relaciones con las mujeres, utilizándola como suplemento de las carencias que su uso evidencia.

Así pues parece razonable que antes de adentrarme en el tema exponga las razones que me hacen estar especialmente sensibilizado ante el mismo.

En primer lugar quiero exponer que escribo estas páginas desde mi condición de enfermera. $\mathrm{Si}$, no me he equivocado en la utilización del género, aunque bien hubiera podido utilizar el de enfermero. Pero da la casualidad de que formo parte de un colectivo eminentemente femenino y en el que la denominación internacionalmente aceptada para referirse a sus profesionales es la de enfermera. Estoy bien seguro de que si se hubiera presentado una mujer como médico o abogado nadie, o muy pocos, hubieran reparado en el detalle, no se habrían arqueado las cejas como señal de sorpresa o de una presumible falta tipográfica, ni suscitado sonrisas de complicidad y se consideraría como algo totalmente normal. Así pues no sé por qué ha de extrañar que yo me presente como enfermera. Sí, soy enfermera. Enfermera macho, si ustedes quieren, pero enfermera. Al igual que existen cebras macho, abejas macho o panteras macho. No sé qué manía existe ahora en eliminar los genéricos (sean del género que sean) para referirse a la condición de las personas utilizando de forma torpe y reiterativa las fórmulas de ciudadano / ciudadana, compañero / compañera, enfermera / enfermero como paradigma o reafirmación del no-sexismo, pasando a convertirse en un acto de violencia gramatical.

Hecha esta aclaración de identidad pasaré a hacer una descripción que en principio puede resultar pueril y fuera de lugar pero que considero necesario hacer para que se den cuenta del por qué de mi identificación en contra de la violencia de género o doméstica.

A lo largo de la historia y a pesar de sus indudables valores y aportaciones ha sido considerada como inferior. Siempre se ha considerado que lo que hacía no tenía valor ni importancia a pesar de que resultaba imprescindible hacerlo. Ha sabido siempre gestionar, organizar, planificar, ejecutar y evaluar su actividad con gran eficacia siendo asumido el éxito por otros que se han considerado como los verdaderos artífices de lo que no necesariamente han realizado. Ha sido constantemente descalificada, menospreciada y humillada haciéndole adoptar roles de sumisión, simplicidad, compasividad y displicencia. Cuando ha querido desarrollarse se le ha impedido o dificultado para evitar que lograse situarse al nivel de los que siempre se han considerado superiores. Ha sido utilizada para alcanzar fines particulares y una vez alcanzados ha sido olvidada. Ha sido falsa, grotesca e irónicamente alabada a la vez que sutil e intencionadamente ignorada, ridiculizada y utilizada en condición de su sexo. Ha sido considerada una oportunista, manipuladora e intrusista por querer alcanzar igual dignidad y reconocimiento social. Ha tenido que soportar los errores por otros cometidos no pudiéndose defender en igualdad de condiciones. Ha tenido que demostrar lo que a otros se les daba por supuesto $\mathrm{Ha}$ tenido que perder su identidad para asumir la que otros han querido otorgarle. Ha sido apartada, aislada y excluida de los foros en los que se hablaba de ella. Y a pesar de todo, ha sido capaz de adaptarse a las necesidades que se pre- 
sentaban. Ha sabido dar respuesta a los problemas que se le planteaban. Ha logrado hacerse un lugar en la sociedad basándose en el esfuerzo. Ha logrado recuperar su identidad. Se ha incorporado en el ámbito científico e investigador,.... estoy hablando de la Enfermería, es decir de la mujer, de la feminidad, de eso que ahora hemos dado en llamar género. Y evidentemente no he venido a hablarles de la Enfermería, pero desde mi condición de enfermera en una profesión eminentemente femenina ésta ha sufrido y aún sigue sufriendo los mismos inconvenientes, dada su feminidad, que cualquier mujer. Es decir, sumisión, incomprensión, anulación de identidad... es por ello que desde la subjetividad que se puede sospechar desde mi posición masculina - que no machista -, pero con la objetividad del feminismo que asumo como miembro de mi profesión "estoy, como dice Rosa $\mathrm{M}^{\mathrm{a}}$ Alberdi, por un feminismo de la igualdad ya que creo que los hombres debemos concienciarnos y luchar por nuestro derecho a desarrollar valores femeninos, sin sentirnos disminuidos ni discriminados" por todo lo cual considero estar en disposición de opinar sobre la violencia doméstica entendida como la ejercida desde el poder mal entendido, en razón a ser de uno u otro sexo, para querer alcanzar lo que se ha considerado hasta hace muy poco como justo, razonable, justificable y perdonable. Y así mismo me permitirá hacer una valoración del papel que los profesionales sanitarios en general y las enfermeras en particular pueden jugar en la promoción, prevención, detección y actuación ante la violencia doméstica.

Alcanzado pues este punto me parece razonable poder exponer cual es mi particular y por lo tanto subjetivo punto de vista con relación al tema en el que se combinan dos problemas de salud de gran trascendencia como son el alcoholismo y la violencia doméstica.

Para empezar tengo que expresar mis dudas con respecto a la relación causa efecto que se trasluce del título del artículo. Sin negar que evidentemente el alcoholismo puede actuar, o de hecho actúa, como un factor potenciador o de riesgo de la violencia éste no siempre puede ser considerado como desencadenante de la misma. Pues no todos los alcohólicos son violentos o potenciales agresores ni todos los agresores son alcohólicos. Así pues, entiendo, no se debe relacionar de forma directa alcoholismo con violencia pues estaríamos cometiendo una victimización secundaria de estos enfermos. Teniendo en cuenta, por otra parte, que son muchas las mujeres que como consecuencia de la violencia que sufren caen en el alcoholismo no convirtiéndose automáticamente por este hecho en agresoras o violentas. Hecha esta consideración como necesario planteamiento de base para poder abordar la cuestión desde una perspectiva real, entiendo que sí que puede resultar interesante hacer una valoración de en qué forma el alcoholismo y la violencia se potencian entre sí.

\section{ALCOHOLISMO Y VIOLENCIA}

Es evidente la interrelación entre alcohólicos y los que con ellos conviven, en la que se fragua una compleja urdimbre de disgustos, fracasos, tensiones, emociones... que finalmente lo convierte todo en negativo, rechazando la evidencia, tanto el propio afectado como la familia, y resistiéndose a relacionar la conflictividad, con la ingesta de alcohol por los potentes motivos socioculturales que hacen de las bebidas alcohólicas una droga normalizada.

Los hijos, se ven forzados a asumir papeles de adulto precoz, y aunque pueden ser víctimas de violencia física, lo más grave y frecuente es la negligencia emocional, o sea, no existe comunicación con el afectado, no se obtiene apoyo del alcohólico y se sienten desamparados como personas, en especial cuando la madre ha iniciado su deriva hacia el alcohol.

En principio se adopta la actitud de comprender / entender durante un largo periodo de tiempo, "el cambio de carácter y de humor" del alcohólico y a su vez se realizan intentos de ayuda sacrificándose a sí mismo. Dadas las aparentes "mejorías" y sucesivos recrudecimientos conflictivos, este intento, orientado de una manera emocional desde la "normalidad" del familiar conduce a que se convierta en cómplice de la enfermedad y contribuya a perpetuarla, sin ser plenamente consciente de ello. Cuando existe o se ha establecido una relación, más o menos confusa, entre bebida y conflicto, intenta compartir parte de los apuros, conflictos, etc. del alcohólico para que este pueda resolver la 
problemática que se supone le inducen a beber, dado que el afectado no es capaz de parar de una manera continuada a pesar de haber verbalizado sus deseos frustrados de autocontrol. En el fondo intenta controlar al afectado, procura percibir la problemática emocional del alcohólico en cierta medida como suya y pretende asumir parte de las supuestas responsabilidades del adicto sin comprender que no son suyas, con lo que muchas veces "ayuda a la enfermedad y no a la persona enferma". Se neurotiza porque a pesar de sus esfuerzos no es capaz de controlar al bebedor, que a su vez no controla la bebida. Existen roces, choques, malestar, ansiedad frente al fracaso. La desesperanza, el coraje, la angustia - ansiedad, la frustración y el resentimiento inician su andadura en la pareja o en otros miembros de la familia o del núcleo doméstico. Es la relación frente a la impotencia de no poder resolver un conflicto en el que se siente y se encuentra implicado y que en el fondo evalúa como "moral", de ahí el calificativo de "vicio". En ocasiones se culpabiliza de su incapacidad para encontrar una manera de tratar "convenientemente" a un ser querido, a pesar de recurrir a amenazas, manipulaciones y de adoptar un claro rol de mártir con eventuales agresiones. Existe una clara violencia que según los niveles socioculturales se expresará como "tortura psicológica" o daños físicos.

En general la posición y el papel de la mujer en la sociedad actual, a pesar de haber superado en muchos aspectos los roles tradicionales, sigue siendo más proclive a desarrollar una relación como la descrita con su pareja alcohólica que el varón.

Por otra parte cuando acuden a los servicios sanitarios mujeres, madres de familia o jóvenes de ambos sexos con supuestos "trastornos de la personalidad", cuya pareja o padre se puede sospechar que padecen un alcoholismo, se niegan a abordar su conflicto dado que aún no se han presentado en los ámbitos laborales / profesionales, sociales o de salud somática evidencias de su trastorno suficientemente alarmantes para el paciente alcohólico.

En un gran número de estos casos, la familia o la pareja no se resiste al impacto de una convivencia profundamente alterada por las conductas del paciente, y el coalcoholismo adquiere dimensiones de psicopatología compleja. En otras la actitud de la familia o de la pareja pueden evidenciar que exista una vida paralela, o que el paciente habite un hogar roto de hecho, en el cual se tolera, quizá porque aún contribuye económicamente al sustento familiar o simplemente porque existe un pacto, no escrito ni explícito, de tolerancia hacia aquel "bulto" del medio familiar con el que no se cuenta ya para nada. En esta situación la profundización del proceso, dependerá de la suplencia de roles firmemente establecida y en ocasiones, sólo la aparición de una enfermedad alcohólica secundaria claramente somática puede cambiar el horizonte de una paz fundada transitoriamente en la convivencia formal.

En múltiples ocasiones, la complejidad de esta relación, provoca situaciones de violencia física en las que factores bilaterales del alcohólico y de la reactividad anómala de los familiares constituyen factores que si se hubieran detectado y se hubieran podido realizar medidas preventivas y de resolución hubieran evitado una relación que socioculturalmente tiene el perfil de peligrosamente masoquista.

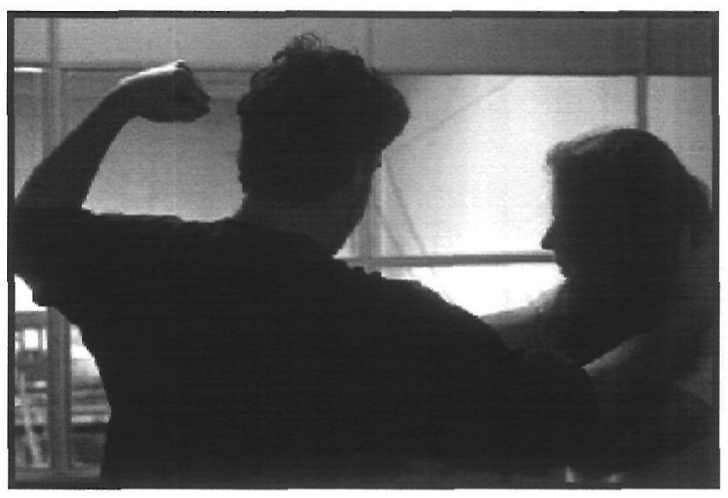

\section{VIOLENCIA DOMÉSTICA}

En cuanto a la violencia doméstica, su consideración como fenómeno privado ha propiciado que durante siglos se considerara, primero un derecho del marido y algo normal, $y$, posteriormente algo que "desgraciadamente" sucedía en algunos hogares pero que formaba parte de la vida privada de las parejas y en lo que por tanto no había que intervenir. De hecho, son muchos los análisis que coinciden en señalar que la consideración de la supuesta "privacidad" de la violencia doméstica es uno de los factores que subyacen al hecho de que las víc- 
timas no denuncien y de que éste continúe siendo un problema "oculto" cuyas cifras reales son casi imposibles de conocer. Por poner algunos ejemplos en este sentido, en España, el informe hecho público por el Ministerio de Trabajo y Bienestar Social sugiere que dos millones de españolas habrían sufrido algún tipo de maltrato por parte de su pareja en algún momento de su vida, y que en la actualidad 600.000 mujeres están siendo víctimas de este delito. Durante el año 2001 ya han muerto en el estado español 59 mujeres y niños a manos de sus parejas y ex - parejas. Ante estos datos resulta imposible negar la existencia de un grave problema social, que va mucho más allá del concepto de "disputas familiares", que afecta profundamente las bases de la convivencia entre hombres y mujeres, poniendo en evidencia substratos alimentados con falsas creencias sobre el significado de masculinidad y feminidad, y sobre la superioridad "natural" de los unos sobre las otras. Hasta 1975, en España, el Código Civil mantenía la potestad marital que autorizaba al marido a corregir a la esposa y obligaba a ésta a obedecerle, estableciendo así tanto la prevalencia del hombre como el derecho de corrección por parte de éste.

En cualquier caso, no corresponde a la justicia penal resolver la complejidad de circunstancias que existen detrás de la violencia doméstica contra las mujeres. Las resoluciones que se dictan en la jurisdicción penal tienen como objeto sancionar comportamientos concretos, sin que pueda pretenderse que su objeto sea el de solucionar las crisis graves de convivencia que puedan presentarse dentro del hogar. Hace falta, por tanto, un amplio debate social que implica un proceso de revisión y crítica con profundidad de muchas de nuestras concepciones sobre el significado de ser hombre o mujer, las normas sociales y de convivencia en las que nos basamos, nuestras expectativas, nuestras aspiraciones, en definitiva el diseño de la sociedad futura que deseamos debe proseguir y ampliarse más. Deberíamos ser capaces de generar como alternativa a la cultura misógina, una cultura de la igualdad, la paz y el respeto a la vida en todas sus manifestaciones y en todos los niveles. Para ello se requerirá el compromiso de todos, tanto individual como colectivamente.

\section{VIOLENCIA DOMÉSTICA Y SISTEMA DE SALUD}

Pero en cualquier caso yo no hablo como experto en violencia doméstica o alcoholismo, pues no lo soy, sino como profesional sanitario y como tal si que puedo hacer una valoración de cual puede o debe ser nuestra aportación, valoración e intervención en temas de tanta trascendencia para la salud individual, familiar y comunitaria.

Para empezar considero que tengo que ser sumamente crítico con la actitud que, por norma general, jugamos los profesionales sanitarios a la hora de abordar no tan solo la problemática concreta que hoy nos ocupa sino al conjunto de la atención ante los problemas sentidos por la población al ser éstos percibidos de forma distorsionada desde una óptica biologicista y poco integral. Minimizamos, cuando no anulamos, la importancia de lo expresado por los pacientes reconduciendo sus manifestaciones hacia problemas físicos generalmente ligados a procesos de enfermedad concretos y aislados de los verdaderos problemas que están generando su consulta, perdiendo la perspectiva necesariamente integral e integradora para analizar los múltiples factores que intervienen tanto en su entorno individual como en el familiar y comunitario, como queda patente en el Objetivo $15^{\circ}$ de la Declaración Mundial de Salud para Todos en el Siglo XXI de la OMS en su $51^{a}$ Asamblea celebrada en Mayo de 1998 que dice: "Resulta importante para los proveedores de Atención Primaria de Salud conocer las circunstancias en las que los pacientes viven: su vivienda, la situación familiar, el trabajo y el entorno físico y social pueden tener una influencia considerable sobre su estado de salud. Salvo que los proveedores de atención sanitaria tomen conciencia de estas circunstancias, los síntomas que aparecen pueden ser mal interpretados y las condiciones existentes pueden ser no reconocidas o ser objeto de un mal tratamiento. El resultado puede ser unos innecesarios procedimientos de tratamiento y de diagnóstico, por lo que se aumentan los costes sin realizar ninguna contribución a la resolución de los problemas reales".

Se contempla, por lo tanto, la salud como ausencia de enfermedad o la enfermedad tan solo como la manifestación de un desequilibrio o dete- 
rioro físico en contra de la definición de salud la OMS, olvidando los aspectos psíquico y social que habitualmente, por no decir siempre, intervienen en cualquier desequilibrio del estado de salud. Pero en cualquier caso y ante el cuestionamiento que sobre esta definición se pueda realizar desde diferentes ámbitos podemos comprobar como desde la perspectiva de la definición hecha por Jordi Gol (a), según la cual "La Salud es aquella manera de vivir autónoma, solidaria y alegre", seguiremos teniendo una percepción reduccionista por parte de los profesionales sanitarios ante los problemas sentidos y manifestados por los pacientes, al actuar como protagonistas únicos en la responsabilidad compartida de la salud, acentuando la individualidad al no participar activa y conjuntamente con los usuarios y confiriendo a todas nuestras actuaciones una rigidez y distanciamiento que no favorecen precisamente la alegría que deberíamos ser capaces de transmitir.

Y si bien es cierto que este planteamiento es general para el conjunto de la población no lo es menos que se hace más evidente en el caso de las mujeres que precisamente por su condición y lo que ha representado histórica y culturalmente en nuestra sociedad, les ha situado como blanco casi exclusivo de muchos de los males que provocan la violencia doméstica y en la que no se puede reducir el problema a un hematoma, una herida, un insulto o una violación, sino que tienen que poderse contemplar los múltiples factores que inciden y desencadenan finalmente en este tipo de lesiones que por sí mismas no tienen mayor importancia que el pronóstico que de ellas se derive. No se puede continuar con la dinámica actual del sistema de salud en el que se atiende a las mujeres de las lesiones físicas, graves o leves, independientemente de su causa, aunque recientemente se esté planteando incluir la violencia doméstica como diagnóstico médico, que es una manera de reconocer en su etiología y consecuencias una dimensión multicausal.

Pero nuevamente nos asalta la pregunta de si la acción de nuestro sistema ha de reducirse a atender a las mujeres sólo cuando alcancen su calidad de víctimas o es posible actuar también en el ámbito comunitario y en el ámbito familiar para combatir los valores y situaciones que sustentan tales prácticas degeneradas, teniendo en cuenta que la familia es la institución de mayor influencia en la socialización del ser humano, siendo la primera escuela de aprendizaje emocional y cognoscitiva a la que se enfrentan los niños; es en el núcleo familiar dónde se recibe el primer modelaje de conducta.(Nevarez, 1996). Por ser el agente de socialización primario, la familia tiene la responsabilidad de proveer a los hijos el desarrollo de actitudes apropiadas y comportamientos considerados importantes por la sociedad (Steinmetz, 1977). La familia se debate entre ser red de sostén económico y emocional, entre otros; y el ser instrumento de violencia entre sus miembros (Silva y otras, 1990). Lamentablemente, es la familia el contexto donde se encuentran los más altos niveles de interacciones violentas (Strauss, 1980; Steinmetz, 1977).

Así pues, lo verdaderamente importante, lo realmente preocupante, lo necesariamente abordable es la situación o situaciones, el ambiente, el entorno, la relación, el miedo, la baja autoestima, el control, la impotencia, la frustración, el aislamiento, la indefensión... que se producen como consecuencia de agresiones, físicas, psíquicas o sexuales, en el entorno doméstico y que son manifestadas de manera casi siempre encubierta con descripciones difusas de depresión, agotamiento, malestar general, etc. que habitual y automáticamente son medicalizadas como ya expresó el criticismo de Illich a la industria médica.

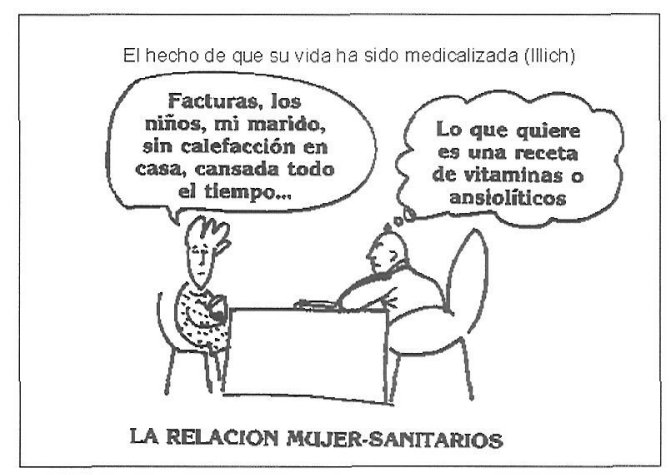

(a) Definición realizada por el médico Catalán Jordi Gol i Gorina en el transcurso del Congreso de médicos y Biólogos de habla catalana celebrado en Perpinya en el año 1975. 
Esta deficiente comunicación genera en la paciente mayor aislamiento, desconfianza y agravamiento de su estado, no tan solo de la salud entendida como malestar físico, sino de todo lo que envuelve a su situación, lo que provocará una cronificación del problema en su conjunto y situarla en un callejón sin salida al comprobar que no son percibidas sus necesidades reales y sentidas. En este sentido podemos destacar los datos aportados en el transcurso del XI Congreso de Enfermería de Urgencias celebrado en Murcia en el que se abordó el papel de las enfermeras ante la violencia doméstica. Y es que del 10 al $15 \%$ de las mujeres que han muerto víctimas de la violencia familiar y que llevaban una media de siete años de maltrato, habían pasado a su vez una media de 10 veces por un servicio de urgencias sin que se hubiera detectado que sufriera violencia familiar.

Por su parte el profesional sanitario no puede ni debe escudarse en la falta de concreción, o malentendida falta de sinceridad manifestada por la paciente, pues su condición de profesional le tiene que llevar a realizar una valoración integral a través de la observación, siendo lo suficientemente hábil como para percibir lo que sus "síntomas" están escondiendo o de forma solapada están queriendo transmitirnos evitando la victimización secundaria con esta actitud.

Hay que sospechar ante determinadas actitudes de la posible víctima como: temor, mirada huidiza, sensación de vergüenza, sentimientos de culpa, explicaciones vagas, contradictorias o confusas; y en la pareja si se muestra; excesivamente preocupado y solícito o al revés, excesivamente despreocupado, despectivo o irónico. La empatía, la escucha activa, la confidencialidad, la disponibilidad, la transmisión de seguridad y confianza y evitar la emisión de juicios de valor deben conducir a generar un ambiente de confianza que favorezca el que se expresen los sentimientos y las verdaderas razones del deterioro de su salud o como mínimo dejar una "puerta abierta" para que sepa que puede hacerlo cuando quiera.

Hay que tener en cuenta que una mujer desde que empieza a ser víctima de la violencia por su pareja hasta que ella misma se hace consciente y se atreve a comunicarlo a aquellas personas con las que se relaciona pasa mucho tiempo. Pasan años también hasta que se decide a comentarlo con su médico o su enfermera (Mc Cauley, 1998). Dice Leininger que "quien necesita cuidados primero busca a personas de su entorno (familiares, amigos, no profesionales), y solo se busca la asistencia profesional si se empeora o teme la muerte" (Leininger, 1978).

Sin embargo, los malos tratos (que en principio suelen ser emocionales y sexuales), tienen muy pronto repercusiones en la salud de las mujeres, de manera que casi desde el principio aparecen síntomas psíquicos o físicos o empeoran los existentes, por lo que las mujeres acuden a sus Centros de Salud (OMS, 1998; Heise , 1999). Pero, en la mayoría de las ocasiones, los síntomas aparecen como desligados de la situación vital que la mujer está viviendo, tanto para el profesional como para la propia mujer. Existen barreras psicológicas y culturales que dificultan que se hable del tema, de las que resaltamos por su importancia: en relación con las mujeres - el negarse a sí mismas lo que está pasando, la culpa, el miedo a la reacción de los demás, etc. y en relación con los profesionales la falta de tiempo y la carencia de formación en este campo (Mc Cauley, 1999; Saller, 2000).

A nuestros Centros de Salud acuden también los hombres que ejercen violencia, solicitando asistencia por sus problemas de salud. Les conocemos pero como no sabemos cómo trabajar con ellos, ni existen lugares de derivación para su tratamiento, no abordamos el tema. De esta forma, contribuimos con nuestra actitud a la invisibilización de la violencia.

Por todo ello no podemos, los profesionales, seguir manteniendo actitudes que favorezcan el despiste de signos de violencia que no harán más que conducir a situaciones extremas o irremediables.

\section{VIOLENCIA DOMÉSTICA Y ENFERMERAS}

Pero este análisis crítico de la situación no tiene que hacernos caer en el desánimo sino más bien todo lo contrario. Es por ello que me parece oportuno aportar las que considero son razones fundadas para expresar el importante papel que juegan las enfermeras, fundamentalmente en Atención Primaria, para poder dar respuesta al problema 
planteado y que ratifica, desde su posición nada sospechosa, la OMS en la Declaración Mundial de Salud para Todos en el siglo XXI donde dice: "...en el núcleo de la Atención Primaria debe estar una enfermera de salud familiar bien formada, que proporcione un amplio asesoramiento en materia de hábitos de vida, asistencia familiar y servicios de atención domiciliaria a un número limitado de familias...".

Las enfermeras en el dilema cuidar - curar están mucho mejor preparadas que otros profesionales sanitarios para superar el aparente fracaso de la tecnología ante este tipo de problemática. Su mayor accesibilidad, su preparación más enfocada a la atención integral, su permanente contacto con la población... les sitúa como piezas fundamentales para la detección de este tipo de problemas al realizar valoraciones alejadas de la especialización por órganos o sistemas o por patologías concretas, centrándose, sin embargo, en la observación, detección y priorización de problemas y en la planificación de cuidados relacionados con los múltiples factores que intervienen en el proceso de la salud. En los que la enfermedad se constituye como un elemento más, pero no en el único ni el más importante, de su amplia actuación profesional. Contemplando a los individuos tanto en su vertiente individual como en la familiar y comunitaria al pasar de la consulta al domicilio, y del domicilio a la comunidad. Asumiendo, además, el papel fundamental de coordinar los diferentes recursos existentes para, interrelacionándolos entre sí, poder dar en cada momento la respuesta más adecuada a través del trabajo conjunto con el individuo que le permita adquirir la capacidad autónoma y responsable del autocuidado, lo que le reportará una mayor autoestima.

Son muchas las Sociedades Científicas y Asociaciones de Enfermería que se están preocupando y actuando de una manera activa en el problema de la violencia doméstica. Así podemos destacar las posiciones adoptadas a este respecto por el Consejo Internacional de Enfermeras (CIE) (b), el abordaje realizado por la FAECAP (Federación de Asociaciones de Enfermería Comunitaria y de Atención Primaria) (c), entre otras.
La mujer maltratada, que en general está aislada socialmente, acude a nuestros Centros de Salud, que es casi el único lugar donde su pareja le deja acudir libremente, aunque no suelen expresar una demanda explícita de ayuda (OMS; 1999; Mc Cauley, 1999), pero en el que las enfermeras pueden detectar signos de alarma o de petición de ayuda a los que dar respuesta. Por ello, los Centros de Salud son un lugar privilegiado y un dispositivo crucial para la prevención, detección precoz, captación e intervención inicial con las mujeres maltratadas (y también con las niñas y niños testigos o sometidos a maltrato, y con los hombres que ejercen violencia).

Se dan en nuestra Comunidad condiciones para que sea posible lo que hoy es ya una de las lecciones aprendidas en las actividades para erradicar la violencia: que éstas se extiendan a todo el sistema de salud ( Heise, 1999). Uno de nuestros puntos fuertes es que hay Centros de Salud, en cada pueblo, en cada barrio.

Bien a través de las Consultas de Enfermería, bien a través de los diferentes programas de promoción y prevención, bien a través de las visitas domiciliarias las enfermeras pueden detectar múltiples problemas a los que poder dar respuesta por ellas mismas o con la colaboración del resto de profesionales del Equipo.

Y si importante es la actuación directa con las mujeres para prevenir y detectar la violencia doméstica y el alcoholismo, no lo es menos la que se debe llevar a cabo para promocionar actitudes no violentas, de convivencia e igualdad a través de intervenciones de Educación para la Salud en la escuela que son desarrolladas en el ámbito escolar y que suponen una de las principales actividades comunitarias de las enfermeras. En este sentido me parece oportuno dar a conocer un estudio realizado en el Estado de Guerrero (México) en el que se pone de manifiesto la necesidad de tales actuaciones aunque evidentemente hay que destacar las diferencias de cultura, desarrollo etc. que sin ser comparables cuanto menos nos tienen que hacer pensar en el problema de la desigualdad de géneros.

\footnotetext{
(b) Declaración de Posición del CIE adoptada en el 2001. (c) En las II Jornadas Nacionales de la FAECAP, Madrid mayo 2001.
} 


\section{ESTADO DE GUERRERO}

\section{INFORME DE LA CONSULTA ESTATAL} POR LOS DERECHOS DE LAS MUJERES

Mujeres Guerrerenses por la Democracia, A.C.

Comité de Defensa Popular, A.C.

Sindicato Académicos de Trabajadores de la Universidad Autónoma de Guerrero

Fecha: 3 y 5 de marzo, 2000

Participantes: 1.081 (598 niñas y 483 niños)

Mesas: 22

Lugares: 6 municipios de la entidad: Chilpancingo, Acapulco, Tlapa, Copanatoyac, Xochistlahuaca y San Marcos.

\section{NIÑAS Y NIÑOS}

1. ¿Las niñas tienen derecho a relacionarse y jugar con los niños?
SI (992)
NO (27)
NO SE (41)

2. ¿Las niñas tienen el mismo derecho que los niños de ir a la escuela?
SI $(1,045)$
NO (25)
NO SE (11)

3. ¿Consideras que las niñas, como las jóvenes, sólo sirven para ser mantenidas?
SI (692)
NO (349)
NO SE (40)

4. ¿Las niñas deben ser maltratadas, ignoradas o ser menos que los niños?
SI (714)
NO (330)
NO SE (27)

5. ¿Crees que cuando una pareja está junta, el hombre es dueño de su mujer?

$$
\text { SI (762) NO (255) NO SE (63) }
$$

Pero una cuestión es que las enfermeras se configuren como las profesionales que, posiblemente, en mejor disposición estén para abordar la violencia doméstica - desde la perspectiva de la promoción, prevención y detección - en el ámbito sanitario y otra bien diferente es que se den las condiciones adecuadas para que este tipo de intervención se produzca. Me explico. La violencia doméstica y el alcoholismo con ser dos problemas de indudable importancia no han sido incorporados en el ámbito sanitario como tales, al estar siendo abordados más desde su vertiente sociológica y psicológica, sien- do atendidas sanitariamente tan solo las consecuencias puntuales derivadas de las mismas, que muchas veces ni tan siquiera llegan a relacionarse con la violencia doméstica como ya hemos visto. Y con ser importantes las aportaciones realizadas desde la sociología y la sicología éstas se producen tan solo en los casos que o bien ya han desembocado en graves problemas y que han sido denunciados por las propias mujeres o que como consecuencia de su gravedad han salido a la luz pública. Así pues nos encontramos con un porcentaje muy pequeño de detección de violencia doméstica al no haberse interiorizado como un verdadero problema de salud y al no tener previsto de forma normalizada qué hacer y cómo cuando acude una mujer maltratada a los Servicios de salud (Centros de Salud, Servicios de Urgencia extra o intrahospitalarios). Es decir no está protocolizada la intervención sanitaria más allá de la elaboración de un parte de lesiones en caso de lesiones físicas. Por lo tanto hace falta una intervención urgente en este sentido de tal manera que los profesionales sanitarios se conciencien de la importancia de su actuación para abordar de forma integral este problema y puedan tener las herramientas y la información adecuadas para poder informar, actuar y derivar de manera eficaz.

Uno de los graves problemas, a mi modo de ver, estriba en la separación de competencias existente actualmente entre el ámbito social y el sanitario que conduce en muchas ocasiones a que los problemas se queden en terreno de nadie o bien se produzca una lucha de intereses derivada de esta separación. Se hace necesaria e imprescindible la actuación socio - sanitaria desde una perspectiva de interrelación efectiva y eficaz en la que no primen más los protagonismos particulares de cada una de las partes que el interés o bien común de las afectadas. No estoy poniendo en duda los esfuerzos llevados a cabo a través de servicios de tanta importancia como los servicios Mujer 24 horas, los centros de Protección a la Mujer, el Servicio de Atención a la Mujer (SAM), los Centros Infodona... que sin duda han supuesto un gran avance, pero que por sí solos no van a ser capaces de abordar el problema en toda su magnitud tal como se desprende de los datos con los que se cuenta. Se hace imprescindible, pues, un acerca- 
miento, coordinación, información, formación y sensibilización de todos los agentes sociales y sanitarios para a través de una adecuada red de comunicación y derivaciones poder canalizar la diferente información recogida que permita dar una respuesta rápida, eficaz y uniforme a las mujeres.

No se trata tan solo de que se les informe o se les ofrezca denunciar. Muchas de ellas no desean que su situación sea conocida y no desean denunciar a su pareja, lo que desearían es que él cambiara; muchas de ellas saben que si él lo sabe puede tomar represalias; saben que no disponen de protección efectiva; tienen miedo de que su familia se rompa. No desean salir de su casa, con sus hijos, como si fueran delincuentes, e irse a una casa de acogida. Muchas veces no tienen recursos económicos para hacer frente a su mantenimiento y al de sus hijos si se separan de su pareja. Tampoco tiene presente el círculo de la violencia en el que casi siempre las mujeres maltratadas están inmersas, que tiene momentos muy malos, pero momentos de calma, de reconciliación...

No se tiene en cuenta que muchas veces la violencia a la que la mujer está sometida está escondida, incluso para ella misma. Aceptar que una misma es víctima de maltrato es un "shock" importante; la mujer maltratada y los profesionales somos miembros de esta sociedad y tenemos prejuicios contra ellas: "son mujeres de bajo status", "algo habrán hecho",.. y tantos otros.

Por otra parte la Administración en su conjunto y los gestores sanitarios y sociales en particular tienen una gran responsabilidad a la hora de abordar esta problemática. Se deberán arbitrar las medidas tendentes a alcanzar la comunicación necesaria que permita desarrollar programas conjuntos de actuación, delimitando claramente los canales de derivación - intervención, dotando convenientemente a los diferentes centros para su actuación, capacitando a los profesionales sanitarios a través de Formación para adecuar la atención sanitaria a las necesidades de las mujeres con un enfoque preventivo y en el que se aborden también las necesidades de los profesionales que trabajan con mujeres maltratadas o con alcohólicos. Asegurando la coordinación institucional como uno de los requisitos fundamentales para el cam- bio. Desarrollando guías en las que se especifiquen los lugares, personas que los atienden, programas que se realizan, población que abarcan... y difundirlo convenientemente para constituir una Red que permita a su vez la adecuada y periódica comunicación a través de encuentros en los que abordar los problemas, experiencias, expectativas... de todos los profesionales implicados.

Los profesionales sanitarios y sociales, por su parte, deberán asumir la responsabilidad que les corresponde actuando de manera multidisciplinar y evitando protagonismos ligados a corporativismos, implicándose en la solución de los problemas detectados a través de una adecuada información y de una eficaz derivación. Registrando la violencia doméstica como problema de salud al igual que se hace con muchos otros factores de riesgo y enfermedades en las Historias Clínicas -En un estudio de un Centro de Salud, las tasas de detección de mujeres víctimas de violencia, aumentó de ninguna, a un $12 \%$, simplemente con una pregunta añadida en la hoja de la Historia Clínica (Freund, 1996)-. Confeccionando registros de morbilidad que nos permitan conocer la prevalencia de las mujeres maltratadas. Desarrollando líneas de investigación que permitan conocer la situación real de la violencia doméstica. No se puede continuar pensando que lo que sucede o acontece en el entorno familiar entra dentro de la privacidad y que por lo tanto no se puede o debe intervenir.

Por otra parte y aunque pueda parecer economicista, no se puede desdeñar el grave problema de costes que supone la violencia doméstica. La OMS cita que son superiores a los accidentes de tráfico, las violaciones y otros actos de violencia conjuntamente. Según el Banco Mundial 1 de cada 5 días laborables que pierden las mujeres por razones de salud es por violencia doméstica. Se calcula que el coste es de 1.600 millones de dólares en Canadá y de 10.000 millones en EEUU. Los costos indirectos pueden superar ampliamente los costos directos según explica Mayra Buvinic, jefa de la Unidad del Programa de la Mujer en el Desarrollo del Banco Interamericano de Desarrollo.

El ámbito sanitario, por sí mismo, es un lugar extraño, hostil y relacionado con la enfermedad que no favorece el acercamiento, la actitud abierta y la confianza, por lo que deberá tenderse a trans- 
formar esta percepción a través de la actuación y la actitud de los profesionales, generando espacios abiertos, exentos de barreras y con amplia información sobre los servicios que oferta. Los centros de Salud no pueden quedar reducidos a lugares de encuentros esporádicos, puntuales y sin continuidad, debiendo, por otra parte, extender su actuación a la comunidad a través de visitas domiciliarias, intervenciones comunitarias, actividades grupales, etc.

La población debe tener una referencia clara de cuales son los profesionales a los que dirigirse con una asignación concreta, clara, precisa y mantenida en el tiempo tanto de médico como de enfermera, tal como se describe en el Objetivo $15^{\circ}$ en su apartado 6.2.2. de la Declaración Mundial de Salud para Todos en el siglo XXI de la OMS en su $51^{\mathrm{a}}$ Asamblea mundial celebrada en Mayo de 1998, en el que dice: "El hecho de atender a una población específica mejora la relación entre los profesionales sanitarios y dicha población. Una característica que contribuye en gran medida a proporcionar la amplia gama de servicios descritos hasta ahora es que cada médico y cada enfermera de salud familiar deben atender a una población específica, definida como un área de captación geográfica o definida mediante la inscripción en una lista. En ambos casos, es importante también que las personas sean capaces de elegir libremente a un profesional al que puedan elegir como "propio" entre la lista de profesionales que ejercen en la zona... Por otra parte, la continuidad de la atención sanitaria queda garantizada, ya que cada persona es seguida de manera continua por el mismo profesional sanitario".

De poco servirá que las enfermeras sean profesionales idóneos para abordar el problema de la violencia doméstica si se sigue con una asignación de población inconcreta, difusa y desconocida por la población. La confianza no se podrá alcanzar desde planteamientos organizativos alejados de las necesidades de la población para acercarlos a los de otros grupos o colectivos internos que para nada favorecen las actuaciones comentadas.

Es importante que la comunidad sea consciente de los recursos con los que cuenta y a los que puede recurrir y se empiecen a eliminar los estereotipos, a los que tan acostumbradas han estado las mujeres, en relación con las enfermeras. Las enfermeras están capacitadas para desarrollar una actividad autónoma a la vez que interdisciplinar alejada de la que habitualmente se le relaciona y por lo tanto se puede y se debe exigir esta aportación.

Quisiera que mis palabras se entendiesen no como una defensa a ultranza, desde mi condición de enfermera, de este colectivo sino como una llamada de atención a la población para que sepan valorar en su justa medida las posibilidades que tales profesionales pueden aportar a un tema de tanta trascendencia como el de la violencia doméstica y el alcoholismo.

\section{EXPERIENCIA EN EL ÁREA DE SALUD 17}

Pero lo apuntado hasta ahora quedaría en un cúmulo de buenas intenciones, sino aportase alguna experiencia que avalase lo dicho. Es por ello que me van a permitir que de forma breve les exponga un ejemplo de lo que actualmente se viene realizando en el Área de Salud 17 de Atención Primaria en la que trabajo y que sin tratarse de un programa específico contra la violencia doméstica si que está centrado en la Mujer.

Desde hace más de dos años se viene desarrollando el programa del climaterio o de la menopausia en la totalidad de Centros de Salud del Área. Dicho programa está dirigido a las mujeres climatéricas. La tipología de mujeres que participan en el citado programa tiene unas características que hacen del mismo no tan solo una intervención sobre la menopausia.

Se trata de mujeres que por su edad (entre 45 65 años) no tienen trabajo retribuido, han visto desplazado, habitualmente, su centro de atención al ser sus hijos mayores y en la mayoría de los casos haber abandonado el núcleo familiar o ser totalmente independientes de los cuidados maternos (síndrome del nido vacío). La relación de pareja sufre a su vez importantes cambios ante esta nueva situación. La autoestima de las mujeres suele disminuir al no sentirse útiles y su sexualidad sufre modificaciones importantes.

La posibilidad de incorporarse a un grupo de mujeres que van a poder compartir experiencias similares, que van a aprender a convivir con su nueva situación, que les va a permitir romper el 
círculo vicioso en el que se han visto incluidas y la posibilidad de sentirse de nuevo valoradas, les va a reportar un aumento considerable de la autoestima.

Un grupo multidisciplinar compuesto por matronas, enfermeras, fisioterapeutas, trabajadores sociales y médicos intervienen en el programa haciéndoles partícipes directas de su situación a través de diferentes actividades.

Es en el inicio o incorporación al programa a través de las entrevistas personales con las matronas y/o enfermeras cuando es más fácil detectar posibles indicios de problemas de salud como violencia doméstica que en muchos casos han venido sufriendo desde hace muchos años, al establecerse un ambiente de confianza y de comunicación. Se trata muchas veces de situaciones que consideran normales al no haber conocido otro ambiente o cultura que la que arrastran de su educación como mujeres.

A través de las intervenciones que a lo largo del programa se desarrollan rompen su aislamiento, el grupo facilita relaciones de amistad y redes de apoyo, desarrollan habilidades de comunicación, escucha, expresión de sentimientos, intercambio de ideas. Aprenden la relación entre iguales, diferentes formas de solucionar conflictos, de tomar iniciativas.

Destacar por ejemplo que en un reciente estudio realizado por los profesionales de enfermería en el Área 17 con mujeres incluidas en el programa y presentado en las Jornadas de Enfermería celebradas en mayo de este año, se obtuvo que un $42 \%$ de ellas refieren tener problemas en su relación de pareja, que un $45^{\circ} 7 \%$ no se siente valorada ni apoyada en su entorno familiar, que solo un $46^{\prime} 9 \%$ de las mujeres expresan tener un buen nivel de apoyo social y que un $50^{\prime} 6 \%$ manifiestan necesitar algún tipo de apoyo respecto a su nivel de ansiedad - depresión. Con ser preocupantes los porcentajes reseñados hay que destacar la importancia de que estas mujeres, a través de las actividades grupales que se llevan a cabo con los profesionales sanitarios, hayan manifestado sus necesidades sentidas y no siempre expresadas y que a través de programas como el reseñado se puedan incluir actuaciones dirigidas a la detección e intervención sobre la violencia doméstica.
Pero si importante es el hecho de que las mujeres logren expresar sus necesidades y problemas no lo es menos el que sean capaces, por ellas mismas, una vez concluido el programa de mantener el grado de autoestima alcanzado. Pues uno de los riesgos que puede tener este tipo de intervenciones es el de que las mujeres se hagan dependientes del sistema y de sus profesionales para seguir manteniendo los logros alcanzados o de que se retorne a la situación inicial al concluir el programa. Es por ello que, a través de la coordinación con los diferentes recursos comunitarios (ayuntamientos, instituciones privadas, servicios sociales, sociedades, asociaciones...), se establecen estrategias para que continúen de forma autónoma con las actividades grupales iniciadas y aprendidas durante el programa, como uno de los objetivos básicos de toda actuación enfermera. Así podemos destacar que se han constituido ya diferentes grupos de mujeres que desarrollan de forma periódica y mantenida diferentes actividades (ejercicio físico, reuniones, viajes...) que facilitan el deseado cambio de calidad de vida en estas mujeres de forma autónoma pero teniendo una referencia clara de los profesionales sanitarios a los que acudir en caso de necesidad y con los que siguen manteniendo cierto grado de participación, que no de dependencia, en algunas de las actividades.

Ejemplos como el expuesto, sin embargo, no deben quedar como excepciones a lo que debe incorporarse como general actuación de los profesionales sanitarios que deben entender que problemas como la violencia doméstica o el alcoholismo, entre otros, son tan importantes como la diabetes, la hipertensión arterial o la depresión que además deben ser valorados en toda su dimensión física, social y psicológica para evitar que queden reducidos a un diagnóstico médico lo que supondría la segura pérdida de información sobre las necesidades reales que tales manifestaciones esconden.

Por último me gustaría que todo lo apuntado no quedara enmarcado en la teoría de la DUALIDAD DEL DISCURSO de Manuel Amezcua, según la cual "la formulación de un hecho, que puede ser un ideal o un proyecto fundamentado, siempre se ve compensada por estrategias contradictorias que son fruto de la reinterpretación interesada que hace el sector fuerte" (Amezcua, 1999:7-9). 
Hay quienes todavía cuestionan programas específicos para la mujer por entender que es otra forma de aumentar la desigualdad entre géneros, pero lo bien cierto es que son precisamente las mujeres las que sufren las consecuencias de una sociedad en la que el miedo a las ideas de los demás y la poca fe en las propias conducen a situaciones de violencia tal como apunta Antonio Fraguas (Forges). Es por ello que se debe seguir trabajando en este sentido pues cuando los pacíficos pierden toda esperanza, los violentos encuentran motivos para disparar (Harold Wilson).

\section{BIBLIOGRAFÍA}

- Alberdi Castell, RM (1997); "Mujeres enfermeras en España. Una historia de márgenes". Revista Rol de Enfermería n 223, pp. 64-70 (Artículo revista).

- Freixa i San Feliu, F (2000); "La reconsideración de las experiencias adversas de los que conviven con un alcohólico/a como recurso socio terapéutico: Un hito a asumir, la codependencia". Editorial Revista Española de Drogodependencias Vol 25 n $^{\circ} 3$ (Editorial revista).

- Barceló Martínez,R, Rosales Amaris, M (1996); "Factores de riesgo asociados a la disfuncionalidad familiar. Barrio ciudad Modesto. Barranquilla, Colombia 1996", Publicación electrónica disponible en http://infomed.uach.cl/relasafa/Tema3.htm (Publicación electrónica) $14 / 11 / 01$.

- Santos de Silva, MR (1996); "Rescatando el conocimiento sobre la familia y el alcoholismo".Publicación electrónica disponible

http://infomed.uach.cl/relasafa/Tema3.htm (Publicación electrónica) 14/11/01.

- Bosch Fiol, E, Ferrer Pérez,VA (2000); "La violencia de género: de cuestión privada a problema social"; Revista intervención Psicosocial. Revista de igualdad y calidad de vida. Vol. 9 n 1, pp. 7-19 (Artículo de revista)

- Informe del Defensor del pueblo sobre violencia doméstica contra las mujeres. Datos estadísticos (2001). Publicación electrónica disponible en:

- http://mys.matriz.net/mys03/especial/especial_003_08.htm (Publicación electrónica) 14/11/01.

- Posada Kubissa, L (2001); "Discurso jurídico y desigualdad sexual: reflexiones para un debate sobre la violencia sexual". Publicación electrónica disponible en: http://www.nodo50.org/mujeresred/violencialuisa_posada.html, (Publicación electrónica) 16/11/01.

- OMS, Oficina Regional Europea de la OMS
(1998); "HEALTH21: an introduction to the health for all policy framework for the WHO European Region. European Helth for All Series, N 5, 1998.

- Mc Cauley, J. et al (1998); "Inside Pandora's Box. Abused women's Experiences with Clinicians and Health Services". J G Intern Med, pp. 549-555 (Artículo revista).

- Wesley, RL; "Teorías y modelos de Enfermería", México 1997, McGraw-Hill Interamericana: pp.120-127 (capítulo de libro).

- OMS/OPS "Violencia contra la mujer. Un tema de salud prioritario" (1998). Washington D.D. Junio, 1998.

- Heise L Ellsberg, M. Gottermoeller M (1999) Ending violence against women. Population reports, Series L, $N^{\circ} 11$. Baltimore, Johns Hopkins University School of Public Health. Population Information Program, 1999.

- Saller, PR, Taliaferro, E (2000); "Reconocimiento y prevención de la violencia doméstica en el ámbito sanitario". Cedecs, Barcelona (libro).

- OMS, Oficina Regional Europea de la OMS (1998); "HEALTH21: an introduction to the health for all policy framework for the WHO European Region. European Health for All Series, No 5, 1998.

- Blanco Prieto, P, Abril Navarro, V (2001); "Propuestas de las Asociaciones de mujeres para un plan integral contra la violencia hacia la mujer en la Comunidad de Madrid"; Consejo de la mujer, 16 de febrero 2001. Publicación electrónica disponible en: http://www.fadsp.org/mujer3.htm (Publicación electrónica) $18 / 11 / 01$.

- Balance de la consulta por los derechos de la mujer realizada en Guerrero (Mexico) (2000). Publicación electrónica disponible en :http://www.laneta.apc.org/ $\mathrm{mmm}$ /guerrero.html (Publicación electrónica) 16/11/01

- Freund, KM, Bak SM, Blackhall L. (1996) ; "Identifying domestic violence in primary care practice". J Gen Intern Med, 11 pp. 44-6 (Artículo revista).

- Informe del Defensor del pueblo sobre violencia doméstica contra las mujeres. Datos estadísticos (2001). Publicación electrónica disponible en: http://mys .matriz.net/mys03/especial/especial_003_08.htm 14/11/01.

- OMS, Oficina Regional Europea de la OMS (1998); "HEALTH21: an introduction to the health for all policy framework for the WHO European Region. European Health for All Series, No 5, 1998.

- Galiana Gómez de Cádiz, MJ, Doménech Bernabéu, I, Chulvi Alabort, V, Flores Alvárez, JC, Bustamante Belda, M, Grau García, MJ, Durá Albert, J, Mira Galván, R; (2001) "Perfil de calidad de vida de las 
mujeres climatéricas de los Centros de Salud de Petrer y Monòver". Comunicación presentada en las XII Jornadas de Interrelación de Enfermería en Aspe (Alicante), mayo. Publicada en el Libro de Comunicaciones y Ponencias editado por el Consejo de Enfermería de la Comunidad Valenciana (CECOVA): pp. 99-102 (Libro).
- Amezcua Martínez, M (2000); Conferencia Inaugural del II Congreso de Enfermería Comunitaria, Mollina, Málaga, Junio 2000. "Enfermería y Atención Primaria: Luces y sombras ante el nuevo milenio". Publicación electrónica disponible en: http://www.asanec.org/Congreso/Enfermeria\%20y\%20AP.htm (Publicación electrónica) 18/11/01

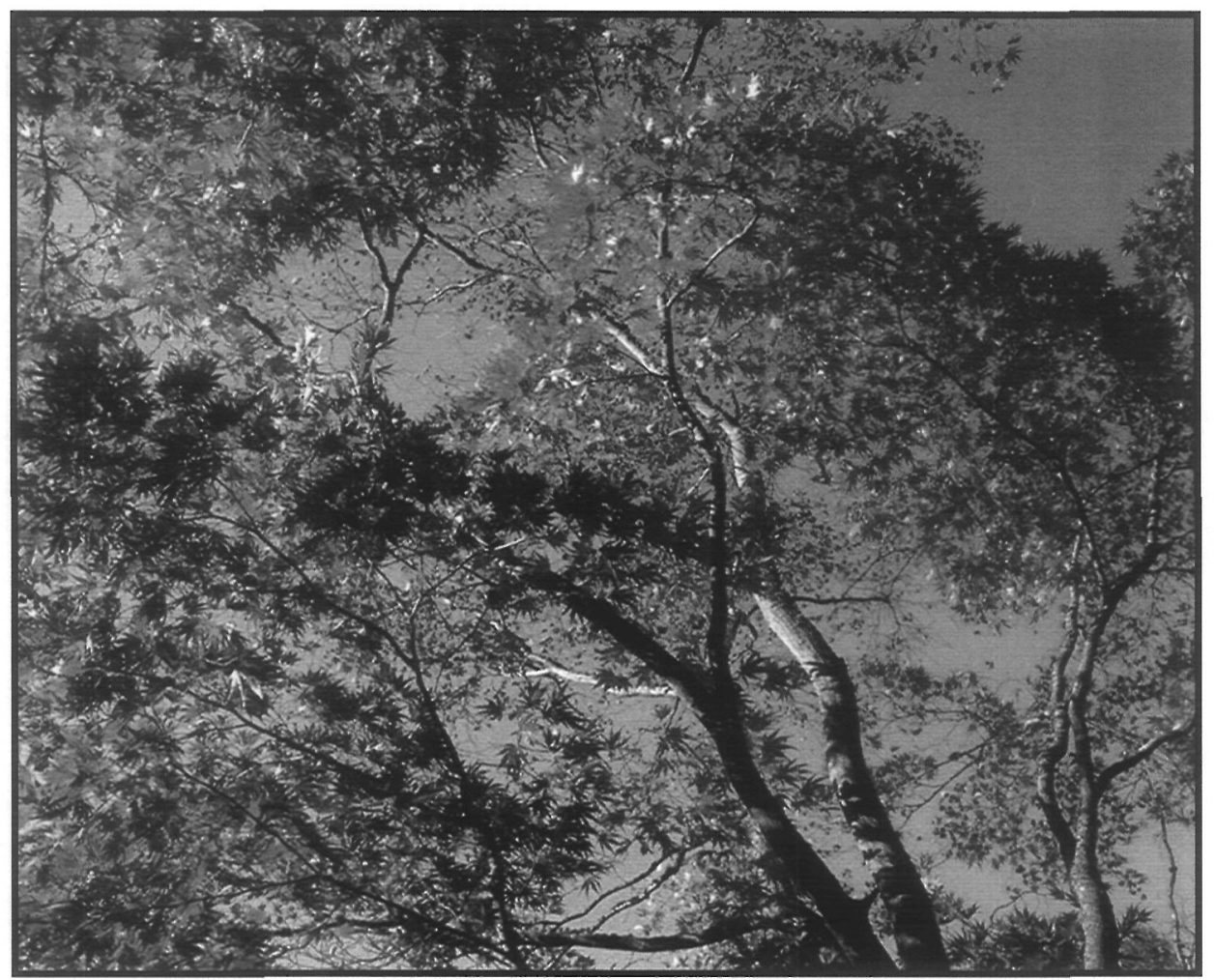

